\title{
A Simple Method for Measuring Adenosine Triphosphate in Acute Cholangitis Patients to Determine the Need for Emergency Biliary Drainage
}

\author{
Tsuyoshi Mishiro ${ }^{1,2}$, Kenichi Kishimoto ${ }^{1}$, Wataru Hamamoto ${ }^{1}$, Yuumi Cho ${ }^{1}$, \\ Hironobu Mikami ${ }^{1}$, Yukihiro Ikuta ${ }^{1}$, Noritsugu Yamashita ${ }^{1}$, Ichiro Moriyama ${ }^{2}$, \\ Norihisa Ishimura ${ }^{2}$, Syuichi Sato ${ }^{2}$, Shunji Ishihara ${ }^{2}$, \\ Shingo Ishiguro $^{3}$ and Yoshikazu Kinoshita ${ }^{2}$
}

\begin{abstract}
:
Objective Acute cholangitis is occasionally life-threatening and requires immediate treatment. For the management of acute cases, globally accepted diagnostic criteria and the use of severity grading, as defined in the Tokyo Guidelines 2018 (TG18), are recommended. This study was performed to explore the association between acute cholangitis and the level of adenosine 5'-triphosphate (ATP) in blood as determined with a simple measurement method.

Methods Twenty-three consecutive patients admitted for acute cholangitis and 14 healthy individuals were enrolled. Based on the TG18, the patients were categorized according to the degree of severity as Grade I, II, or III. We measured the amount of ATP in blood samples using a bioluminescence meter and evaluated the correlation with the degree of severity.

Results The ATP/total hemoglobin (tHgb) level showed a significant decline in association with an increase in severity, as that in the healthy controls was $236.60 \pm 8.10$ and in the Grade I, II, and III groups was $238.56 \pm 6.98,186.88 \pm 7.62$, and $154.60 \pm 11.01$, respectively $(\mathrm{p}<0.01)$. While no significant difference was observed between the healthy controls and Grade I patients $(\mathrm{p}=0.649)$, there was a statistically significant difference between Grade I and Grade II $(\mathrm{p}<0.01)$ in the ATP/tHgb level. According to a receiver operating characteristic analysis, the area under the curve for ATP/tHgb, used as an index for predicting the need for emergency biliary drainage (Grade II, III cases), was the highest among various examined factors.

Conclusion The present novel measurement method was found to be simple to perform and useful for detecting acute cholangitis patients with a low ATP level who may require emergency biliary drainage.
\end{abstract}

Key words: cholangitis, Tokyo Guidelines 2018 (TG18), adenosine 5'-triphosphate (ATP)

(Intern Med 58: 3213-3217, 2019)

(DOI: 10.2169/internalmedicine.2569-18)

\section{Introduction}

Acute cholangitis is a clinical condition in which acute inflammation occurs within the bile duct due to biliary stenosis or blockage (1-3). The risk of mortality is high in affected patients not treated with antibiotic therapy or when biliary pressure is not immediately reduced with an appropriate method, so a reliable diagnosis and assessment of the severity of cholangitis is crucial for predicting potential mortality.

For the management of acute cholangitis, referral to glob-

${ }^{1}$ Department of Gastroenterology and Hepatology, National Hospital Organization Hamada Medical Center, Japan, ${ }^{2}$ Department of Gastroenterology and Hepatology, Faculty of Medicine, Shimane University, Japan and ${ }^{3}$ Department of Cardiac and Vascular Surgery, National Hospital Organization Hamada Medical Center, Japan

Received for publication December 24, 2018; Accepted for publication June 17, 2019

Correspondence to Dr. Tsuyoshi Mishiro, tmishiro@med.shimane-u.ac.jp 
ally accepted diagnostic criteria and the use of severity grading defined in the Tokyo Guidelines 2018 (TG18) are recommended (4-6). Notably, the updated severity assessment criteria are considered to have made it possible to accurately select the optimal timing for performing biliary drainage for Grade II (moderate acute cholangitis) or Grade III (severe acute cholangitis) cases at the time of the diagnosis. However, because of restrictions related to various examination items and a lack of medical staff at night in the emergency room, it is sometimes difficult to perform grading according to TG18. As a result, determining whether or not emergency biliary drainage should be implemented for a particular case can be problematic.

Adenosine 5'-triphosphate (ATP), a central component of energy storage and metabolism in vivo, is involved in several cellular processes, including respiration, biosynthetic reactions, motility, and cell division $(7,8)$. Critically ill patients with multiple organ failure and septicemia show decreased mitochondrial activity and ATP production. Indeed, blood ATP depletion has been identified in children with influenza-associated acute encephalopathy (9). Although released ATP cannot be accurately analyzed due to rapid degradation, the cellular ATP level, including the blood cell ATP level, is easily measured with a bedside procedure that uses the sum of ATP production and ATP degradation and/or release $(10,11)$.

In the present study, we examined the correlation between the blood ATP level evaluated at the bedside with a simple measurement method and the degree of severity in acute cholangitis patients diagnosed based on the TG18 criteria.

\section{Materials and Methods}

\section{Ethics statement}

The study protocol was approved by the ethics committees of Shimane University School of Medicine and National Hospital Organization Hamada Medical Center. Written informed consent was obtained from each subject prior to enrollment. When it was difficult to obtain a signed consent form from a patient because they were unconscious, we explained the procedures to the family members and obtained their written informed consent prior to enrollment.

The study was carried out in accordance with the Declaration of Helsinki and registered with the University Hospital Medical Information Network clinical trials registry (UMIN 000031569).

\section{Patients}

We evaluated 23 consecutive patients admitted for acute cholangitis from May 2016 to September 2018. In addition, the study also included 7 healthy individuals $<75$ years old [healthy controls (HC)] and 7 healthy elderly individuals $\geq$ 75 years old [healthy elderly controls (HEC)], all of whom were free from any acute or chronic illness. As for the cause of cholangitis in the patient population, 9 patients were af- fected by common bile duct stone occlusion, and 14 had cholangitis with an unknown etiology. Based on the TG18, the patients were divided by the degree of severity into the Grade I (mild acute cholangitis), Grade II (moderate acute cholangitis), and Grade III (severe acute cholangitis) groups. At the time of the diagnosis, the ATP level was determined, and its correlation with the degree of severity was considered.

\section{Blood collection and determination of the ATP level}

Blood was withdrawn from the antecubital vein directly into vacutainer tubes (Becton Dickinson Diagnostics, Tokyo, Japan). After receiving written informed consent from the subject or appropriate family member, the ATP level was determined with a hematology test using the remaining portion of the obtained sample. The blood ATP level was measured using a Lumitester PD-30 ${ }^{\circledR}$ System and LuciPac ${ }^{\circledR}$ PenAQUA kit (Kikkoman Biochemifa, Tokyo, Japan), which are specifically designed to evaluate the amount of ATP within a liquid sample based on bioluminescence using firefly luciferase, with the concentration expressed as number of relative light units (RLU). The kit employs an enzyme cycling method based on a combination of luminescent reactions from firefly luciferase and pyruvate, and orthophosphate dikinase (PPDK). With this method, a given amount of luminescence proportional to the amounts of ATP and adenosine monophosphate (AMP) present is obtained. Firefly luciferase emits light in the presence of ATP and luciferin, and AMP produced from that reaction is converted back into ATP using PPDK, which provides a high but stable amount of luminescence that can be measured. We confirmed that these methods were able to accurately determine the amount of ATP (RLU) using standard ATP solutions (Sigma-Aldrich, St. Louis, USA) (Fig. 1). For this confirmation procedure, autoclaved saline solutions containing $0.01,0.05$, and 0.1 $\mu \mathrm{M}$ of ATP standard powder were prepared. The ATP meters showed a linear relationship between RLU and the actual concentration of ATP. To examine the repeatability of the results, all tests were performed in triplicate. For each test, $100 \mu \mathrm{L}$ of an extracted blood sample was diluted 10,000fold with normal saline solution. Next, a sampling stick from the LuciPac ${ }^{\circledR}$ Pen-AQUA kit was soaked in the diluted sample and shaken gently, according to the protocol supplied by the manufacturer. The bioluminescence produced was then immediately measured using the Lumitester PD-30 ${ }^{\circledR}$ System.

\section{Statistical analyses}

All quantitative values are expressed as the mean \pm standard error. An analysis of variance (ANOVA) using the Student-Newman-Keuls method was performed using the SPSS statistical package, version 24.0 (IBM SPSS Japan, Tokyo, Japan). For this study, a p value $<0.05$ was considered to indicate statistical significance. Receiver operating characteristic (ROC) curves were used to evaluate the diagnostic value of various markers for differentiating acute cho- 
langitis between Grade I and II, including white blood cells (WBCs), platelets (Plt), C-reactive protein (CRP), and adenosine 5'-triphosphate/total hemoglobin (ATP/tHgb).

\section{Results}

The disease severity classification of the 23 patients with cholangitis was Grade I in $8(34.8 \%)$, Grade II in 9 $(39.1 \%)$, and Grade III in $6(26.1 \%)$. Trend testing was conducted using comparisons by an ANOVA, which showed a significant declining trend for the average values of $\mathrm{Hgb}$, Plt, TP, albumin (Alb), and ATP $(\mathrm{p}<0.01)$ in association with an increase in severity grade. In contrast, a statistically significant increasing trend was observed for WBCs, lactate de-

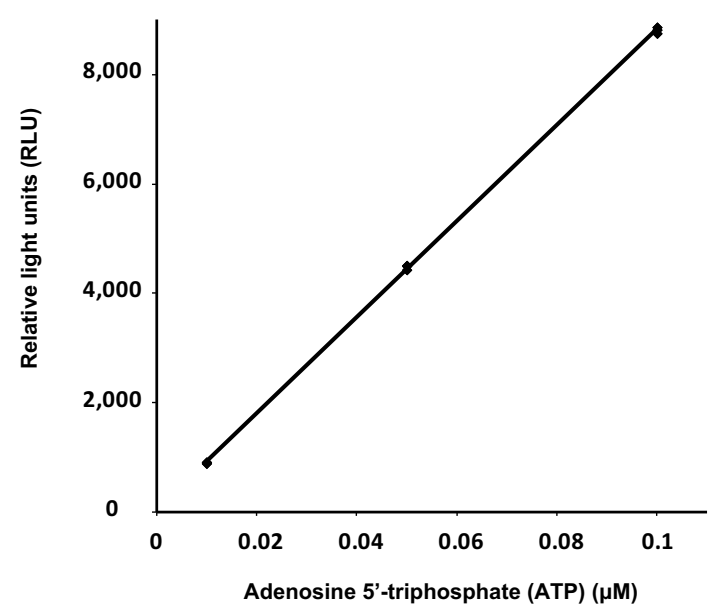

Figure 1. Linearity of ATP readings obtained with ATP luminometer meters (Lumitester PD-30 ${ }^{\circledR}$ System). The ATP luminometer meters used in this study were tested for linearity for reading standard ATP solutions. ATP standard powder solutions $(0.01,0.05,0.1 \mu \mathrm{M})$ were prepared in autoclaved saline solution. The ATP meters showed a linear relationship between RLU and the actual concentration of ATP. hydrogenase $(\mathrm{LDH})$, blood urea nitrogen $(\mathrm{BUN})$, creatinine (Cr) $(\mathrm{p}<0.01)$, Tbil, and CRP $(\mathrm{p}<0.05)$ in association with an increasing grade (Table). There were no marked differences with regard to the gender ratio among the severity grades. Early biliary drainage was performed for one patient with Grade I, nine with Grade II, and four with Grade III. Unfortunately, one of the six patients classified as Grade III died from sepsis-induced organ damage three days after hospitalization.

Blood cells become diluted by transfusion, and a low red blood cell (RBC) count is usually found in patients with advanced disease, resulting in a low blood level of ATP in those who are severely ill (11). Since the major source of ATP in blood is RBCs, the blood ATP levels in the present study were normalized by the level of tHgb (ATP/tHgb). The ATP level (determined by RLU/tHgb) showed a significant decline in association with an increased severity grade (Fig. 2), as that in the HC and HEC groups was $236.60 \pm$ 8.10 and $232.86 \pm 5.35$, respectively, while that in the Grade I, II, and III groups was $238.56 \pm 6.98,186.88 \pm 7.62$, and $154.60 \pm 11.01$, respectively $(\mathrm{p}<0.01)$. While there were no significant differences observed among the HC, HEC, and Grade I groups ( $\mathrm{p}=0.649)$, a statistically significant declining tendency that corresponded with the increase from Grade II to Grade III was noted $(\mathrm{p}<0.01)$. Changes in the blood ATP/ tHgb level in representative patients in the Grade II and Grade III groups after admission are presented in Fig. 3. All of the present patients showed an increase in the ATP/tHgb level in association with clinical symptom improvement.

In the ROC analysis of WBCs, Plt, CRP, and ATP/tHgb, the latter showed the highest value for the area under the curve, which is used as an index for predicting the need for emergency biliary drainage in Grade II and III patients (WBCs 0.819, Plt 0.604, CRP 0.569, ATP/tHgb 0.944) (Fig. 4). Furthermore, the optimal cut-off value for ATP/ tHgb calculated with the Youden index method was 204.49,

Table. Association of the TG18 Severity Assessment Criteria for Acute Cholangitis.

\begin{tabular}{llccccc}
\hline & & HC $(\mathrm{n}=7)$ & HEC $(\mathrm{n}=7)$ & Grade I $(\mathrm{n}=8)$ & Grade II $(\mathrm{n}=9)$ & Grade III $(\mathrm{n}=6)$ \\
\hline Age & $($ years $)$ & $59.71 \pm 5.45$ & $85.57 \pm 1.19$ & $69 \pm 2.74$ & $72.44 \pm 5.13$ & $87.83 \pm 2.10$ \\
Gender & $(\mathrm{M}: \mathrm{F})$ & $5: 2$ & $3: 4$ & $4: 4$ & $5: 4$ & $3: 3$ \\
WBC & $(/ \mathrm{uL})$ & $5,451 \pm 650$ & $5,107 \pm 551$ & $6,721 \pm 748$ & $13,043 \pm 1,764$ & $11,173 \pm 1,049$ \\
$\mathrm{Hgb}$ & $(\mathrm{g} / \mathrm{dL})$ & $14.53 \pm 0.53$ & $12.06 \pm 0.26$ & $12.98 \pm 0.56$ & $11.72 \pm 0.62$ & $11.07 \pm 0.70$ \\
$\mathrm{Plt}$ & $\left(\times 10^{3} / \mathrm{uL}\right)$ & $235.71 \pm 11.21$ & $190.29 \pm 15.90$ & $256.88 \pm 14.74$ & $239.78 \pm 20.61$ & $106.83 \pm 19.31$ \\
TP & $(\mathrm{g} / \mathrm{dL})$ & $6.98 \pm 0.14$ & $6.86 \pm 0.13$ & $6.91 \pm 0.16$ & $6.66 \pm 0.15$ & $5.86 \pm 0.17$ \\
$\mathrm{Alb}$ & $(\mathrm{mg} / \mathrm{dL})$ & $4.37 \pm 0.09$ & $3.90 \pm 0.06$ & $3.78 \pm 0.18$ & $3.27 \pm 0.31$ & $2.78 \pm 0.23$ \\
Tbil & $(\mathrm{mg} / \mathrm{dL})$ & $0.81 \pm 0.19$ & $0.45 \pm 0.07$ & $2.89 \pm 1.29$ & $5.48 \pm 1.68$ & $3.28 \pm 0.72$ \\
LDH & $(\mathrm{U} / \mathrm{L})$ & $191.71 \pm 11.67$ & $194.86 \pm 9.26$ & $227.38 \pm 30.25$ & $489.22 \pm 147.17$ & $369.67 \pm 70.00$ \\
$\mathrm{BUN}$ & $(\mathrm{mg} / \mathrm{dL})$ & $15.92 \pm 1.26$ & $17.39 \pm 1.46$ & $13.25 \pm 1.10$ & $16.79 \pm 1.88$ & $33.42 \pm 11.24$ \\
$\mathrm{Cr}$ & $(\mathrm{mg} / \mathrm{dL})$ & $0.74 \pm 0.05$ & $0.81 \pm 0.07$ & $0.60 \pm 0.04$ & $0.96 \pm 0.10$ & $1.37 \pm 0.44$ \\
$\mathrm{CRP}$ & $(\mathrm{mg} / \mathrm{dL})$ & $0.05 \pm 0.01$ & $0.04 \pm 0.01$ & $3.82 \pm 1.61$ & $5.20 \pm 2.13$ & $8.08 \pm 2.06$ \\
ATP & $(\mathrm{RLU})$ & $3,383 \pm 151$ & $2,848 \pm 95$ & $3,091 \pm 148$ & $2,207 \pm 172$ & $1,692 \pm 115$ \\
ATP/tHgb & $(\mathrm{RLU} / \mathrm{g} / \mathrm{dL})$ & $236.60 \pm 8.10$ & $232.86 \pm 5.35$ & $238.56 \pm 6.98$ & $186.88 \pm 7.62$ & $154.60 \pm 11.01$ \\
\hline
\end{tabular}

WBC: white blood cells, Hgb: hemoglobin, Plt: platelets, TP: total protein, Alb: albumin, Tbil: total bilirubin, LDH: lactate dehydrogenase, BUN: blood urea nitrogen, Cr: creatinine, CRP: C-reactive protein, HC: healthy controls, HEC: healthy elderly controls, ATP: adenosine 5'-triphosphate, ATP/tHgb: adenosine 5'-triphosphate/total hemoglobin, TG18: Tokyo Guideline 2018 


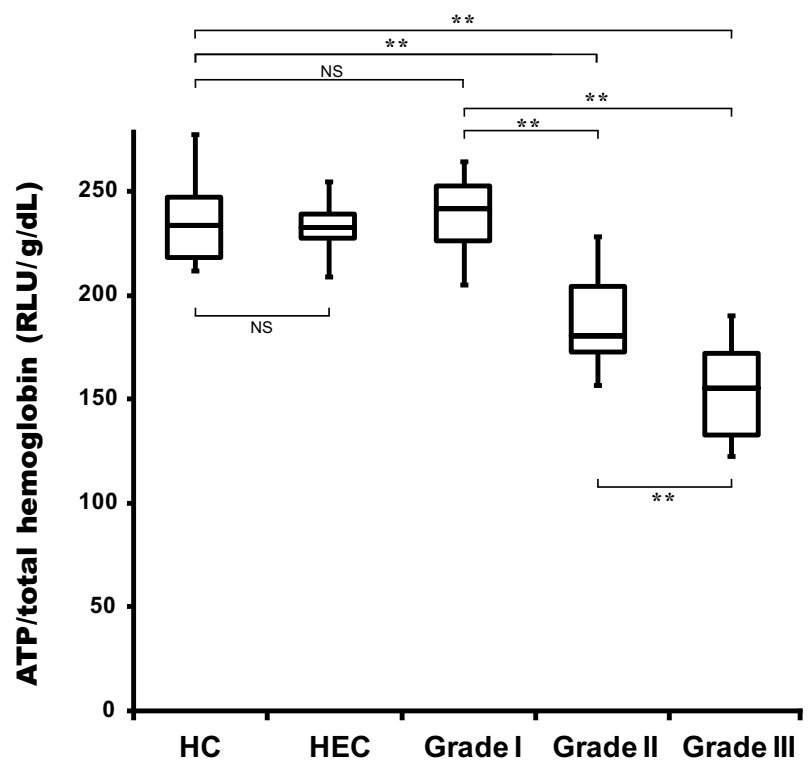

Figure 2. The association of the ATP/tHgb level with the severity assessment criteria for acute cholangitis shown in Tokyo guidelines 2018 (TG18). A statistically significant declining trend was observed for the ATP (RLU/tHgb) level with increasing grade (HC and HEC, 236.60 \pm 8.10 and $232.86 \pm 5.35$, respectively; Grade I, II, and III, $238.56 \pm 6.98,186.88 \pm 7.62$, $154.60 \pm 11.01$, respectively; $p<0.01$ ).

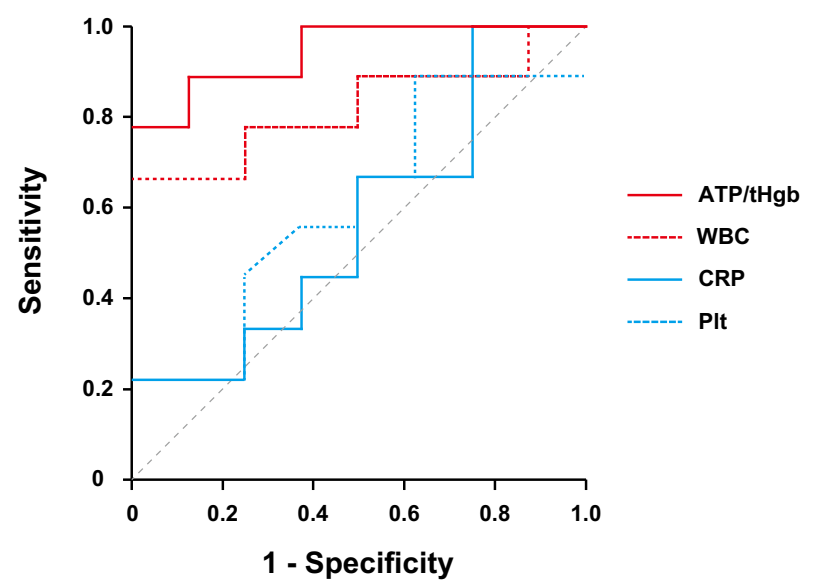

Figure 4. A receiver operator characteristic (ROC) analysis for predicting the need for emergency biliary drainage. ROC curve for the Tokyo guideline 2018 (TG18) severity assessment criteria between Grade I and Grade II. Area under the curve (AUC): white blood cell (WBC) count 0.819 , platelets (PIt) 0.604, C-reactive protein (CRP) 0.569, and ATP/tHgb 0.944.

with a sensitivity of 0.778 and specificity of 1.000 .

\section{Discussion}

Over the past 2 decades, reported rates of mortality associated with acute cholangitis have ranged from $2.7 \%$ to $10 \%$ (12). In order to improve the mortality of affected patients, a method for rapidly determining the degree of severity is needed, so that appropriate action can be taken. With

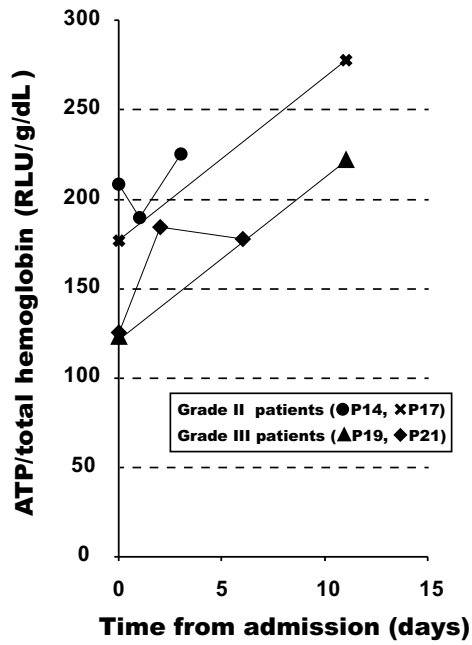

Figure 3. Changes in the blood ATP/tHgb level in representative patients following admission. Shown are serial changes in blood ATP/tHgb from the time of admission according to the severity of the clinical condition in representative Grade II and III patients.

the updated TG18 severity assessment criteria, it is now thought possible to accurately select the optimal timing for biliary drainage in Grade II cases at the time of the diagnosis (4-6). The criteria for determining the degree of severity shown in the updated TG18 were decided based on the WBC count, presence of a fever, age, level of Alb, presence of jaundice, circulatory impairment, consciousness disturbance, respiratory and renal function impairment, hepatic function disorder, and blood coagulopathy. However, because of various restrictions related to the examination items and a lack of medical staff at night in emergency rooms, it can be difficult to accurately perform grading according to the TG18. Therefore, it is occasionally challenging to determine whether or not emergency biliary drainage should be implemented, and the identification of markers that can be easily used to accurately diagnose the presence of sepsis and organ damage in acute cholangitis patients is considered to be extremely important. Ideally, elucidating inflammatory markers that might be useful for diagnosing the degree of severity of acute cholangitis would allow for the establishment of an inexpensive and easy measurement method that is highly sensitive and quick, provides a diagnosis of early stage septicemia, and be correlated with the degree of severity of infection.

Recently, ATP released from damaged tissues has been classified as a potential danger signal, termed an alarmin, because it induces proinflammatory cytokines, although is rapidly degraded within a few minutes by ecto-ATPase (13). Brealey et al. reported that ATP production was reduced in patients in a septic state with complex I dysfunction in the mitochondrial respiratory chain, caused by peroxynitrite and other reactive oxygen substances (14). Although once ATP is released it cannot be accurately analyzed, its cellular level, including blood cells, can be easily determined based on the sum of ATP production and ATP degradation and/or release. 
The blood ATP level tends to be slightly lower in older individuals ( $\geq 60$ years old) than in younger, normal individuals, although the difference is not significant (11), and there is also no significant gender difference in the normal ATP level. However, in the present study, a significant difference in the ATP level was observed between the HC and HEC groups $(3,383 \pm 151$ vs. $2,848 \pm 95$ RLU, $p<0.01)$, although the difference was not significant after correction with $\mathrm{tHgb}$ $(\mathrm{ATP} / \mathrm{tHgb})(236.60 \pm 8.10$ vs. $232.86 \pm 5.35, \mathrm{p}=0.721)$.

Recently, an ATP bioluminescence assay method was developed for use in the food and beverage industry to determine the degree of microbial contamination or cleanliness $(15,16)$. The Lumitester PD-30 ${ }^{\circledR}$ System and LuciPac ${ }^{\circledR}$ Pen-AQUA kit are specifically designed to evaluate microbial contamination of water and other liquid samples by measuring the concentration of ATP (17). We confirmed that those approaches are able to measure relative amounts of ATP (RLU) in standard ATP solutions as well as diluted blood samples, and a distinctive advantage of this ATP bioluminescence assay technique is the ability to rapidly determine the ATP level (within one minute).

To our knowledge, this is the first study to show an association between acute cholangitis and the blood ATP concentration, although it is limited by the low number of patients included in the analyses. Our findings may be helpful for determining the optimal timing for performing urgent biliary drainage using a very simple method. Although we also presented a cut-off value that may be used to determine the need for emergency biliary drainage, the accumulation of more cases is necessary in order to elucidate more detailed factors related to the optimal cut-off value. In addition, we intend to perform a prospective study regarding the correlations of the ATP/tHgb level with the TG18 grading, drainage effect, and prognosis in affected patients.

\section{Conclusion}

The present results suggest that acute cholangitis patients with a low level of ATP require emergency biliary drainage.

\section{The authors state that they have no Conflict of Interest (COI).}

\section{Acknowledgement}

We thank Rika Tohma and Keiko Masuzaki (Shimane University, Japan), and Kozue Katte and Yohko Kawato (National Hospital Organization Hamada Medical Center, Japan) for their assistance, as well as members of our hospital for their helpful advice.

\section{References}

1. Lipsett PA, Pitt HA. Acute cholangitis. Front Biosci 8: s1229s1239, 2003.

2. Lee JG. Diagnosis and management of acute cholangitis. Nat Rev Gastroenterol Hepatol 6: 533-541, 2009.

3. Chung AY, Duke MC. Acute biliary disease. Surg Clin North Am 98: 877-894, 2018

4. Kiriyama S, Kozaka K, Takada T, et al. Tokyo Guidelines 2018: diagnostic criteria and severity grading of acute cholangitis (with videos). J Hepatobiliary Pancreat Sci 25: 17-30, 2018.

5. Miura F, Okamoto K, Takada T, et al. Tokyo Guidelines 2018: initial management of acute biliary infection and flowchart for acute cholangitis. J Hepatobiliary Pancreat Sci 25: 31-40, 2018.

6. Mayumi T, Okamoto K, Takada T, et al. Tokyo Guidelines 2018: management bundles for acute cholangitis and cholecystitis. J Hepatobiliary Pancreat Sci 25: 96-100, 2018.

7. Singer M. Critical illness and flat batteries. Crit Care 21(Suppl 3): 309, 2017.

8. Kozlov AV, Lancaster JR Jr., Meszaros AT, Weidinger A. Mitochondria-meditated pathways of organ failure upon inflammation. Redox Biol 13: 170-181, 2017.

9. Kubota M, Chida J, Hoshino H, et al. Thermolabile CPT II variants and low blood ATP levels are closely related to severity of acute encephalopathy in Japanese children. Brain Dev 34: 20-27, 2012.

10. Crouch SP, Kozlowski R, Slater KJ, Fletcher J. The use of ATP bioluminescence as a measure of cell proliferation and cytotoxicity. J Immunol Methods 160: 81-88, 1993.

11. Chida J, Ono R, Yamane $K$, et al. Blood lactate/ATP ratio, as an alarm index and real-time biomarker in critical illness. PLoS One 8: e60561, 2013.

12. Kimura Y, Takada T, Strasberg SM, et al. TG13 current terminology, etiology, and epidemiology of acute cholangitis and cholecystitis. J Hepatobiliary Pancreat Sci 20: 8-23, 2013.

13. Kobayashi T, Kouzaki H, Kita H. Human eosinophils recognize endogenous danger signal crystalline uric acid and produce proinflammatory cytokines mediated by autocrine ATP. J Immunol 184: 6350-6358, 2010.

14. Brealey D, Brand M, Hargreaves I, et al. Asspciation between mitochondrial dysfunction and severity and outcome of septic shock. Lancet 360: 219-223, 2002.

15. Green TA, Russell SM, Fletcher DL. Effect of chemical cleaning agents and commercial sanitizers on ATP bioluminescence measurements. J Food Prot 62: 86-90, 1999.

16. Omidbakhsh N, Ahmadpour F, Kenny N. How reliable are ATP bioluminescence meters in assessing decontamination of environmental surfaces in healthcare settings? PLoS One 9: e99951, 2014.

17. Fukada T, Tsuchiya Y, Iwakiri H, Ozaki M. Adenosine triphosphate bioluminescence assay for monitoring contamination of the working environment of anaesthetists and cleanliness of the operating room. J Infect Prev 16: 8-13, 2015.

The Internal Medicine is an Open Access journal distributed under the Creative Commons Attribution-NonCommercial-NoDerivatives 4.0 International License. To view the details of this license, please visit (https://creativecommons.org/licenses/ by-nc-nd/4.0/).

(C) 2019 The Japanese Society of Internal Medicine Intern Med 58: 3213-3217, 2019 